\title{
Strates
}

STRATES Matériaux pour la recherche en sciences sociales

$10 \mid 2001$

Villageois et citadins de Grèce

\section{Organisation de l'espace et nouvelles tendances de la ruralité en Grèce}

\section{Guy Burgel}

\section{(2) OpenEdition}

1 Journals

\section{Édition électronique}

URL : http://journals.openedition.org/strates/389

DOI : $10.4000 /$ strates.389

ISSN : $1777-5442$

\section{Éditeur}

Laboratoire Ladyss

\section{Édition imprimée}

Date de publication : 1 mai 2001

ISSN : 0768-8067

\section{Référence électronique}

Guy Burgel, «Organisation de l'espace et nouvelles tendances de la ruralité en Grèce », Strates [En ligne], 10 | 2001, mis en ligne le 31 janvier 2005, consulté le 07 septembre 2020. URL : http:// journals.openedition.org/strates/389; DOI : https://doi.org/10.4000/strates.389

Ce document a été généré automatiquement le 7 septembre 2020

Tous droits réservés 


\title{
Organisation de l'espace et nouvelles tendances de la ruralité en Grèce
}

\author{
Guy Burgel
}

1 La Grèce reste un bon exemple des surprises réservées par le monde contemporain. Une présentation caricaturale, d'ailleurs soigneusement entretenue par le lobby grec à Bruxelles, voudrait que ce petit pays (11 millions d'habitants), longtemps isolé à l'extrémité de la Communauté européenne, soit le plus pauvre de l'Union. En fait, les plus-values de la construction, érigée en sport national, la pluriactivité, répandue dans tous les milieux, le tourisme, grand diffuseur de consommations et de revenus, ont depuis longtemps enrichi bien des Grecs, les transformant en citoyens aisés dans un pays aux finances médiocres. De la même façon, la mondialisation - et la Grèce, nation traditionnelle d'échanges et de migrations, y fut précocement engagée, au-delà de ce que semblait entraîner son niveau économique - devait conduire à l'uniformisation des genres et des espaces de vie, des formes de l'entreprise et des types de sociabilités. C'est le contraire qui est en train de se produire sous nos yeux. Et c'est certainement heureux. Les mêmes processus ne produisent pas les mêmes effets, parce que les histoires sociales et politiques, les inerties et les accumulations, contribuent à différencier durablement les territoires. Les pays reprennent leurs droits à l'heure des organisations supranationales, et le local renforce le global dans l'élaboration d'un monde où les rythmes s'accélèrent en même temps que les espaces deviennent discontinus. Ainsi, la Grèce est certainement le pays européen où, proportionnellement, la métropolisation est la plus forte - la région urbaine d'Athènes avoisine les quatre millions d'habitants - et la ruralité la plus ancrée dans les consciences, les paysages et même la distribution apparente de la population.

2 Ces réalités sociales et spatiales mouvantes constituent autant de mises en garde, que le chercheur doit sans cesse rappeler et se rappeler, à l'égard des approches statistiques. Elles n'interdisent pas pour autant d'utiliser les résultats des recensements grecs pour apprécier les grandes différenciations géographiques du pays et évaluer l'inégale 
rapidité de leurs dynamismes. Il s'agit moins de s'attacher à la véracité des données absolues calculées en général avec une rigueur arithmétique, qui masque, pour un observateur non prévenu, la fluidité des critères de définition, que de révéler à travers elles les grands clivages du territoire, les distinctions majeures entre les groupes et les retournements des temporalités. En Grèce plus qu'ailleurs, analyse statistique et approche sociologique, monographie locale et généralisations prudentes, doivent être conjointement pratiquées, si le chercheur et le politique veulent prendre le risque d'une compréhension raisonnée et d'un volontarisme efficace. Le pays reste un remarquable laboratoire pour les sciences sociales et un bon champ d'expérimentation des directives de l'action, qu'elle soit nationale, ou parée sous l'étiquette technocratique de "politique agricole commune » (PAC). Un bref cheminement dans les campagnes grecques en convaincra aisément.

Des disparités géographiques qui s'accentuent : quatre types de territoires spécifiques1. La montagne

3 Pays de densité démographique moyenne, la Grèce présente de très grands contrastes de peuplement, qui ont eu tendance à s'amplifier au cours des dernières décennies, pour opposer de vastes ensembles vides à des zones de fortes concentrations humaines et économiques. L'exemple n'est pas unique en Europe ( $c f$. la France), mais prend ici une physionomie caricaturale (tableaux 1 et 2, carte 1). Pour s'en tenir aux grandes lignes, une longue échine de basses densités, toujours inférieures à 30 habitants, parfois à 20, au kilomètre carré, parcourt le pays du Nord au Sud, des frontières albanomacédoniennes à l'extrémité du Péloponnèse (nomes de Drama, Florina, Kastoria, Iannina, Thesprotie, Grévéna, Évrytanie, Phocide, Arcadie, Laconie). Terres montagneuses, souvent de hautes cimes (Olympe, Pinde, Parnasse, Taygète), pauvres en hommes et en villes, vouées presque exclusivement à une agriculture et à un élevage extensifs résiduels, elles ont surtout connu pendant le $\mathrm{XX}^{\mathrm{e}}$ siècle une forte hémorragie démographique, qui a achevé d'en faire un territoire désertifié, sinon inhospitalier, de pâturages et de forêts. La décennie 1961-1971 a été ici particulièrement meurtrière, enlevant brutalement un quart, un cinquième de la population. Depuis, l'exode rural s'est assagi, faisant même parfois place à un très petit accroissement au cours de la dernière période intercensitaire (1981-1991). 
Carte 1 : Répartition dela population grecque en 1991 (urbaine, semi-urbaine, rurale)

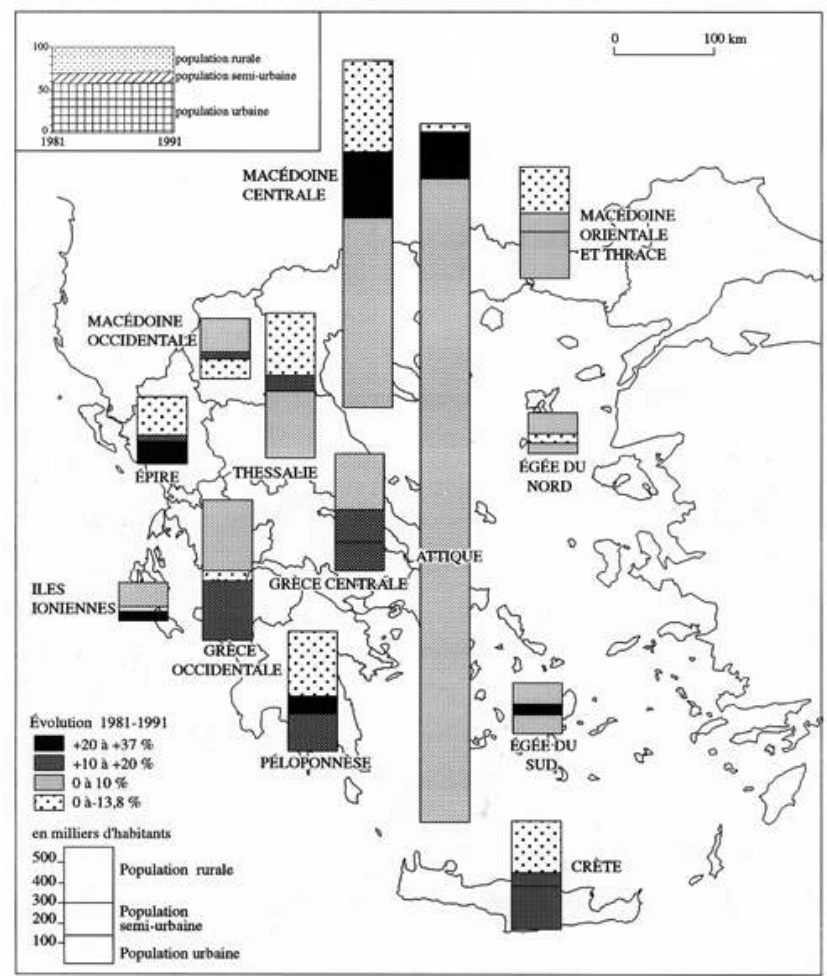

4 Mais ne nous trompons pas sur ces quelques couleurs retrouvées. L'émigration s'est arrêtée parce que le réservoir était tari depuis longtemps. Et le regain démographique, pour réel qu'il soit, et pas purement artificiel par des retours de circonstance le jour du recensement, cache surtout des réinstallations saisonnières ou définitives de retraités, qui n'enrayent pas, loin s'en faut, le vieillissement des populations. Dans ces régions de solitude, sans dire que les politiques nationales ou communautaires sont sans conséquence sur le maintien des activités agricoles et pastorales, le principal problème devient la sauvegarde de l'environnement naturel. Il ne s'agit pas seulement de protéger des valeurs esthétiques, paysagères, végétales ou animales-refuges, pour en faire la ressource hypothétique d'un agrotourisme encore largement à créer, mais de comprendre que la Grèce riche et peuplée dépend aussi des équilibres maintenus dans cet arrière-pays pauvre et peu fréquenté. Érosion des sols, ressources en eau, pour les adductions urbaines, l'irrigation, ou l'énergie hydroélectrique, grandes forêts continues de résineux et d'espèces à feuilles caduques, tout se joue en fait dans cette Grèce du vide, où la désertification humaine fait progressivement reculer les traces de l'anthropisation: les terrasses, qui s'effondrent et accélèrent la descente des terres arables, les champs qui retournent à la friche, les vignes qui deviennent des ronciers. Il n'y a pas lieu devant ces évocations d'avoir des regrets passéistes, qui oublieraient la peine des hommes dans ces contrées dures au travail et chiches au rendement, mais de mesurer les dangers que leur abandon définitif ferait courir au pays tout entier, avant même de penser à promouvoir leurs richesses pour alléger les surcharges touristiques littorales.

Dans cette Grèce continentale de l'intérieur, les petites villes sont relativement rares, dépassant exceptionnellement 20000 habitants (Tripolis en Arcadie), et souvent inférieures à la barre statistique des 10000 habitants (Karpenissi en Évrytanie). Elles 
connaissent en général, sauf cas de récession particulière (la fourrure de Kastoria) une petite progression démographique, même en situation d'isolement géographique. C'est qu'elles concentrent services publics, commerces, possibilités de récréation, et surtout de santé et d'éducation, dont les populations sont de plus en plus en quête. Ce rôle s'est naturellement amplifié sous l'effet de l'accessibilité routière et des mobilités résidentielles saisonnières. C'est une intégration sociale et spatiale à prendre en compte, moins pour développer des activités productrices (lesquelles? et sur quelle base de main-d'œuvre ?) que pour faire de ces petites villes des points d'ancrage et des bases de départ pour la sauvegarde des équilibres naturels montagnards.

2. Le monde insulaire

6 Avec des densités à peine supérieures, des départements insulaires (les Cyclades dans l'Égée, le Lassithi en Crète) paraissent présenter des caractéristiques identiques: densités faibles, recul démographique important dans les années soixante suivi d'une stabilisation. En fait, ici la proximité ou l'immédiateté de la mer change tout. Pour certaines petites îles, l'enclavement peut demeurer plus important, notamment pendant les longs mois d'hiver, que pour bien des régions de montagne, directement reliées au réseau routier. Mais, partout, le littoral, même accidenté, signifie le tourisme estival et balnéaire et une population presque entièrement dévolue, sauf en Crète, aux activités qui lui sont liées : hôtellerie, restauration, commerces de souvenirs, services divers, depuis la location de motocycles jusqu'aux agences de voyages et aux transports en autobus et bateaux locaux.

7 Là encore, alors que souvent plus de la moitié de la population est classée rurale (64 \% pour le département des Cyclades au recensement de 1991), il semble souvent vain de parler véritablement d'une économie agricole réelle, tant l'essentiel des ressources et des rythmes de vie dépend du tourisme et de ses périodicités saisonnières. Pourtant, chacun peut rester ici attaché à son lopin de terre, à quelques têtes de petit bétail, à une production céréalière résiduelle pour nourrir les bêtes, et à quelques cultures arbustives, parfois aussi symboliques que spéculatives (cas de la vigne à Santorin). Le monde insulaire cumule souvent des caractéristiques contradictoires et contrastées : une faible occupation de l'espace qui se transforme pour quelques semaines en sur utilisation des zones littorales en périodes et en zones particulièrement sensibles (l'été, la bande côtière), un médiocre engagement agricole, mais de fortes sollicitations sur l'environnement rural et maritime (effluents locaux de constructions éparpillées et multipliées, de tavernes provisoires, ramassage et traitement des ordures aléatoires, réseaux électrique et téléphonique implantés sans respect de l'impact sur les paysages). Les politiques rurales sont en fait ici - ou devraient être - avant tout d'aménagement et de conservation du domaine maritime et du littoral.

3. Les grandes régions agricoles de plaine

8 Régions de montagne au sens large et monde insulaire - à l'exception de la majeure partie de la Crète - représentent près de la moitié du territoire national, mais ne regroupent qu'environ un dixième de la population permanente de la Grèce et ne constituent qu'une partie infime de son potentiel agricole. Contraction de l'œekoumène, concentration de l'espace agricole utile autour des grandes plaines orientales du pays (Thrace, Macédoine, Thessalie), agglomération exacerbée de l'urbanisation autour de quelques grands pôles (Athènes, Thessalonique, Patras) ont agi dans le même sens: densifier la population sur une faible partie du territoire, additionner les dynamismes démographiques relativement médiocres de la Grèce sur quelques départements 
favorisés, accumuler les richesses agricoles, industrielles et tertiaires sur des régions proches et contiguës (carte 2). C'est décrire le fameux croissant de fertilité et de prospérité qui parcourt la Grèce sur près de 800 kilomètres du nord au sud, de Thessalonique à Athènes, puis de l'est à l'ouest d'Athènes à Patras, sur une largeur qui excède rarement 100 kilomètres, et qui parfois est beaucoup plus réduite (huerta de la côte nord du Péloponnèse). Si l'on ajoute à cet arc du développement hellénique quelques excroissances plus périphériques - la Macédoine orientale et la Thrace littorale, l'Élide dans le nord-ouest du Péloponnèse, la Crète centrale autour d'Iraklion on a résumé les grandes disparités du pays.

Carte 2 : Tendances d'évolution des départements grecs (nomoi) 1961-1991

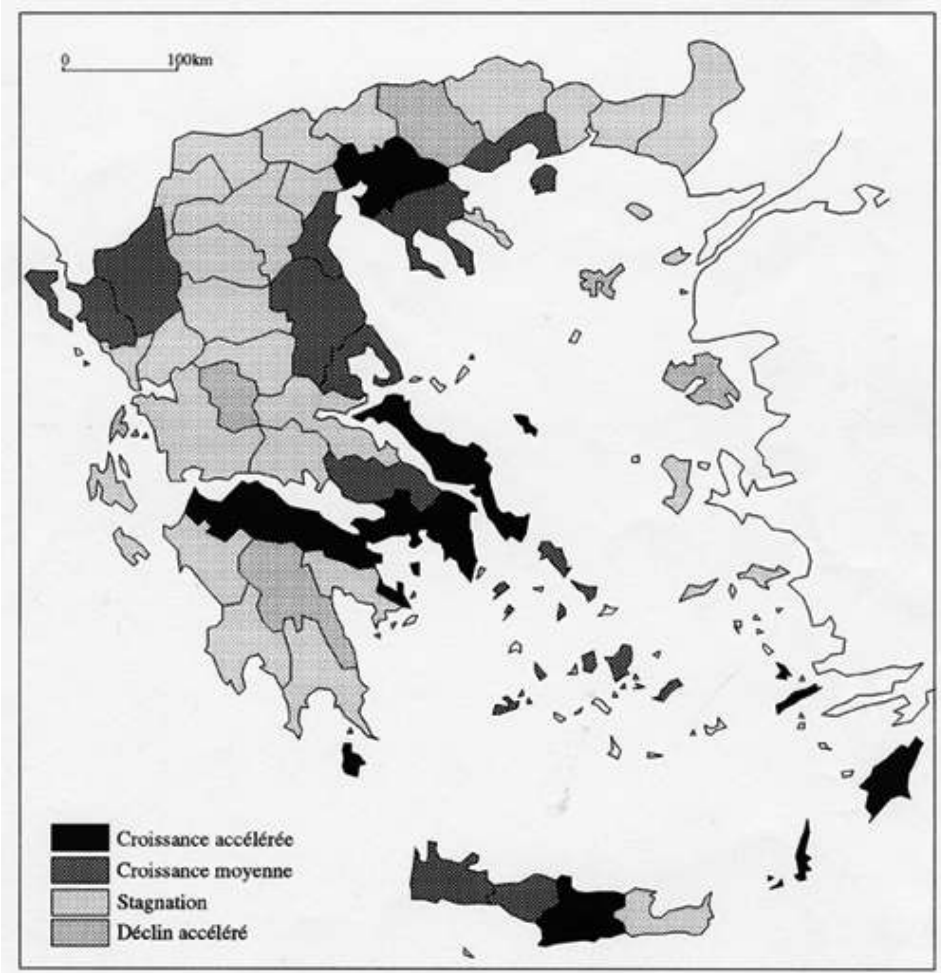

Il faut pourtant y distinguer les régions à prédominance agricole quasi absolue des zones périurbaines, au sens très large du terme, des principales agglomérations. Dans les premières (département de Karditsa et de Trikala en Thessalie, Élide dans le Péloponnèse), la population agricole reste majoritaire, même si on ne lui adjoint pas la population des bourgades semi-urbaines. C'est là que l'économie agricole, notamment autour de la triade coton-blé-tabac, à laquelle il faudrait ajouter la betterave à sucre et une partie de l'élevage bovin, domine toute la vie régionale, y compris celle des centres urbains, préfectures de quelques dizaines de milliers d'habitants, qui regroupent les services administratifs officiels, mais surtout toutes les fonctions de desserte en amont et en aval des productions végétales et animales, et l'exutoire d'une partie de leurs richesses (commerces, investissements immobiliers). Ici, la sensibilisation aux politiques agricoles nationales et communautaires est extrême, comme en témoignent les manifestations paysannes périodiques, qui s'essaient à bloquer les grands axes de circulation du pays, comme à l'automne 1996. Sans négliger les problèmes d'environnement (surabondance de l'utilisation des engrais et des pesticides, irrigation puisant dans les réserves d'eau), l'incidence des réglementations nationales et 
européennes est directe sur un milieu professionnel averti et voué à une agriculture intensive, à qui on a inculqué pendant des décennies la philosophie du rendement, sinon de la productivité. Les régions agricoles de plaine constituent évidemment la pierre angulaire de l'application de la nouvelle politique agricole commune.

4. Les zones périurbaines des grandes agglomérations

10 Enfin, autour des plus grandes villes du pays (Athènes, Thessalonique, Patras, Iraklion, Volos), se développent, ou s'étirent, sur plusieurs dizaines de kilomètres, voire une centaine dans le cas de la capitale multimillionnaire (plus de trois millions d'habitants dans la seule agglomération statistique en 1991), toutes les conditions d'un complexe agro-urbain, où la proximité de la mer, la demande des sociétés locales à fort niveau de vie, et l'afflux du tourisme international, ajoutent encore une utilisation ludique et récréative de l'espace. Constructions résidentielles plus ou moins saisonnières, infrastructures routières et leurs annexes (stations services, aires de parking, surfaces commerciales), desserrement industriel et tertiaire, se mêlent aux installations touristiques (plages organisées, marinas aménagées, hôtels de week-end ou de long séjour) et aux utilisations agricoles plus ou moins spéculatives.

11 La résistance de cette agriculture périurbaine est d'autant plus assurée qu'elle s'articule autour de spécialisations solidement implantées depuis longtemps : vignobles de Pallini en Attique, agrumiculture et vignes à raisin sec autour de Patras, olivettes et vergers de pommiers aux environs de Volos, oléiculture et vignes de la région d'Iraklion. La demande urbaine les complète de son lot habituel d'exploitations florales et horticoles, de production de primeurs et de laitages, sur des unités souvent minuscules, mais que la rentabilité financière justifie. Quand les conditions naturelles et techniques le permettent, il faut encore agrémenter cette concurrence aiguë des utilisations du sol du développement de grandes cultures, ici sur des surfaces importantes : riz, coton, sur les deltas irrigués et remembrés de l'Aliakmon, de l'Axios et du Gallikos, aux portes occidentales même de Thessalonique.

12 On imagine ce que cet encombrement compétitif de l'espace dans un environnement naturel fragile peut entraîner de pressions foncières, d'irrationalités subies, de pollutions graves, de consommation croissante en eau, et d'atteintes plus ou moins irréversibles au patrimoine végétal et marin. Les incendies de forêts estivaux autour de - et quelquefois dans - Athènes, Thessalonique et Patras, sont cycliques, mais ont pris ces dernières années des allures dramatiques : dévastation de la zone boisée de SeichSou surplombant Thessalonique à l'été 1997, tragédies humaines et écologiques dans les faubourgs nord d'Athènes sur les pentes du Pentélique au cours de l'été 1998. Dans un pays qui n'est réputé, ni par l'efficacité de son aménagement, ni par la coordination de sa protection civile, ni surtout par une prise de conscience de la solidarité des problèmes environnementaux, on mesure le chemin à parcourir et l'importance des mesures communautaires à mettre en œuvre. Il s'agit là encore moins de politique agricole que des conditions indispensables pour restaurer un développement rural durable.

$13 \mathrm{Au}$ total, la quadruple typologie qui vient d'être esquissée et dont on a fourni les exemples les plus caractéristiques, à l'exclusion de toute volonté d'énumération exhaustive du territoire, est très loin de recouvrir la diversité et l'hétérogénéité de l'espace rural grec, dont on connaît l'extraordinaire complexité locale. Elle montre pourtant la difficulté majeure de l'application d'une politique agricole nécessairement globalisante et unitaire, quand les problèmes essentiels sont, selon les cas, sinon 
contradictoires, du moins très circonscrits. Tantôt, il s'agit d'abord d'affronter les conséquences de la désertification humaine (espace montagnard, quelques îles isolées). Tantôt, la surcharge littorale saisonnière apparaît la difficulté majeure (domaine insulaire). Tantôt, les concurrences spatiales entre les différents usages du sol sont incontournables (régions urbaines des grandes agglomérations). C'est finalement seulement dans les grandes plaines, essentiellement en Grèce du nord, à proximité immédiate et en contiguïté, mais non en confusion avec le croissant fertile du pays, que les priorités agricoles s'imposent. Ce n'est pas le moindre paradoxe, dans ce pays où l'on signale toujours l'importance du volume de la population rurale, de constater la faible prise d'une politique agricole directe sur un secteur social pourtant vital pour l'équilibre de la nation.

Un exode rural stoppé depuis les années quatre-vingt

Dans ce territoire de diversité, de contrastes et de vives disparités spatiales, il faut souligner très fortement la rupture des années quatre-vingt, attestée par tous les indicateurs démographiques et par leur distribution géographique. Les deux décennies des années soixante et soixante-dix avaient été marquées par une urbanisation exacerbée et un exode rural accéléré. Dans un pays qui connaît, sous l'influence de l'émigration extérieure et de la fécondité assagie, une croissance modérée (+ 1,3 million d'habitants en vingt ans), la seule population urbaine gagne plus de deux millions de citadins, et les campagnes perdent 700000 personnes, passant de $44 \%$ de la population totale à $30 \%$ (Carte 3 ).

Et les pourcentages peuvent être trompeurs sur la brutalité et l'inégale répartition géographique de ces bouleversements considérables de la distribution de la population. Dans les départements (nomes) des très grandes villes du pays (Athènes, Thessalonique, Larissa, Volos, Patras, Iraklion), le nombre des citadins double pratiquement. Sans être de type tiers-mondiste, l'urbanisation grecque favorise, à la taille du pays, la création de très grandes agglomérations, dont la capitale fournit évidemment la démonstration caricaturale. Inversement, certains départements ruraux, insulaires ou continentaux, sont saignés à blanc, perdant de la moitié à un tiers de leur population. Ainsi l'Évrytanie, dans la chaîne du Pinde, voit fuir 15000 de ses 36000 habitants ruraux initiaux, les Cyclades 22000 habitants (sur 77000 en 1961). Il conviendrait souvent d'ajouter à cette dévitalisation des campagnes, le marasme des petites villes qui tentent de les animer : la capitale des Cyclades, Ermoupolis, qui connut des heures de gloire au $\mathrm{XIX}^{\mathrm{e}}$ siècle, stagne désespérément autour de 16000 habitants. À l'opposé, les régions agricoles qui se modernisent et connaissent des débuts souvent flamboyants de spécialisations agricoles, voient leur population rurale se stabiliser ou même augmenter : l'Imathia, autour des vergers de pêchers et de pommiers de Verria et de Naoussa, Piéria, autour du coton et du tabac de Katerini (Macédoine centrale), le département d'Arta (Épire) et la Corinthie (Péloponnèse), autour de l'agrumiculture. On ne répétera jamais assez que ces vingt années furent tournantes pour la réorganisation de l'espace humain et économique de la Grèce, urbain et rural à la fois.

En comparaison, la décennie quatre-vingt, suivie certainement par la décennie quatrevingt-dix (mais rappelons que le dernier recensement de population date de 1991) présente une physionomie plus assagie et des retouches plus que des remaniements, à l'architecture mise en place. Première surprise, dans un pays qui continue à avoir une très lente croissance démographique (9 750000 habitants en 1981, 10250000 en 1991), les populations rurales ont cessé leur déclin, perdant seulement 50000 unités en dix 
ans, pour se stabiliser aux alentours de $28 \%$ de la population totale. La répartition d'ensemble des catégories statistiques reste quasiment immuable : $60 \%$ dans les villes, $12 \%$ dans les bourgades de 2000 à 10000 habitants (population semi-urbaine), le reste dans les campagnes.

Carte 3 : Population rurale en 1991

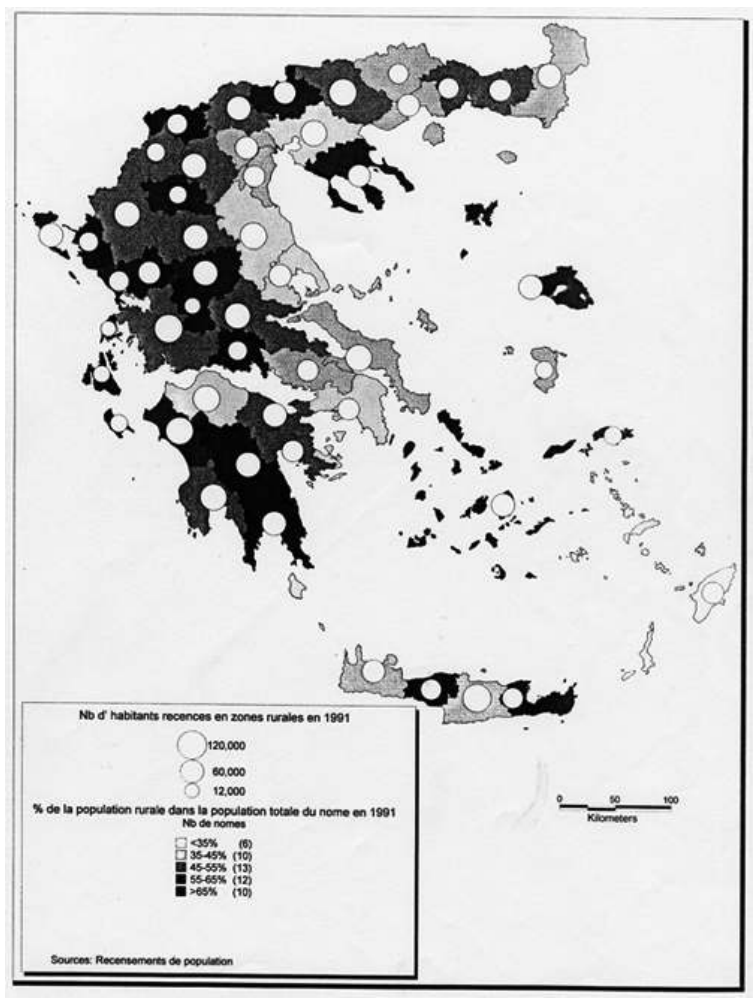

Encore cette stabilité apparente cache-t-elle d'évidentes inégalités géographiques. Rattrapage régional, saturation des très grandes villes, ou urbanisation en profondeur du pays, la croissance urbaine concerne désormais beaucoup plus les villes moyennes que les très grandes agglomérations : Iannina en Épire, Agrinion en Étolo-Acarnanie, Katerini et Kozani en Macédoine, Pyrgos dans le Péloponnèse. De même, la grande périphérie des principales condensations urbaines est touchée par une large périurbanisation (Eubée, Corinthie autour d'Athènes). Cette simple énumération montre que l'urbanisation désormais plus diffuse est sans rapport avec la richesse régionale, notamment agricole, et correspond plutôt à la diffusion de la modernité, à travers et dans la ville.

De la même manière, si la population rurale continue à décroître faiblement dans de grandes régions agricoles (Macédoine orientale et Thrace, Thessalie, et même Crète), elle est en augmentation absolue dans des zones manifestement influencées par l'essor touristique (Îles Ioniennes, Égée du Nord et du Sud), ou le développement de spécialisations agricoles (Grèce occidentale), et évidemment dans les régions des grandes agglomérations notamment en Grèce centrale, autour d'Athènes. La carte 4 montre l'ubiquité de cette reprise rurale - certainement accentuée, ne le cachons pas, par les biais statistiques déjà évoqués - et sa difficile liaison avec une causalité unique : l'Épire littorale, zone pauvre mais profitant du développement du port d'Igouménista, aux portes de l'Adriatique et de l'Albanie, et du tourisme de la charmante baie de Parga 
(Thesprotie), en bénéficie, tout autant que les bords du golfe pagasitique, autour de Volos (Magnésie), les grasses campagnes intérieures de Trikala ou de Béotie, les îles de l'Égée orientale (Lesbos, Khios), sans parler évidemment des Cyclades, paradis touristique. En tout cas, l'hypothèse d'un exode rural actuel important ou d'un potentiel démographique résiduel exagéré dans les campagnes grecques ne repose sur aucun signe statistique objectif.

Carte 4 : Évolution de la population rurale (1981-1991)

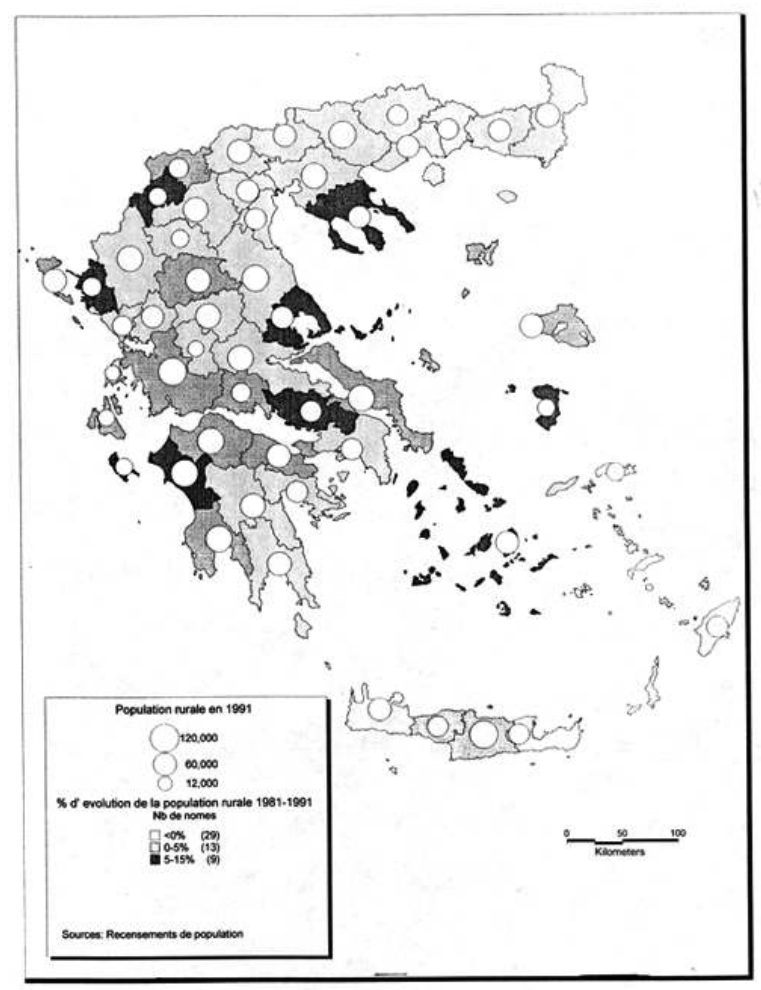

Pour aller plus loin, les causes profondes de ces retournements des temporalités démographiques de l'espace rural hellénique ne sont pas liées uniquement, et même souvent principalement, à la vitalité et aux dynamismes de l'économie agricole, et à l'application des différentes mesures nationales ou communautaires la concernant. Les rythmes fondamentaux sont ailleurs, dans l'ensemble de la société grecque et la place du pays dans le monde. Ainsi, l'exode rural des années soixante et soixante-dix s'explique plus par l'ouverture de la Grèce sur la modernité et le monde extérieur (urbanisation, industrialisation, début de la consommation de masse, appel migratoire de l'Europe occidentale) que par une crise interne des campagnes, qui étaient alors plus caractérisées par la pauvreté et le sous-équipement que par la mévente des produits agricoles. De la même façon, le retournement des années quatre-vingt traduit avant tout des modifications considérables des genres de vie dans les espaces ruraux (l'automobile, la télévision et la vidéo, les transports rapides, les équipements ménagers et les infrastructures publiques) avant même la bonne santé de l'économie. Tout cela n'est pas propre à la Grèce, mais comme toujours y a été assimilé et adopté avec une étonnante rapidité et flexibilité.

S'ajoutent certainement à cette influence majeure de la diffusion de la modernité dans l'espace grec - retard des campagnes, puis envahissement massif et brutal - d'autres 
causes plus directement liées à la conjoncture économique ou démographique. Il faut ici rappeler le retournement du comportement migratoire quasi séculaire de la Grèce à la fin des années soixante-dix, déjà évoqué plus haut. De pays traditionnel d'émigration, d'abord balkanique - sous la domination turque, une partie de l'élite du Phanar est d'origine hellénique -, puis transocéanique (États-Unis, Canada, Australie), enfin européenne, vers l'Allemagne fédérale notamment, la Grèce est devenue un pays d'immigration, plus ou moins comptabilisée. Affaiblissement de la demande de maind'œuvre dans une Europe de l'Ouest qui s'essouffle économiquement et se tourne vers des gains de productivité, retours de travailleurs migrants nationaux, ouverture de l'Europe de l'Est et balkanique après la chute du communisme, besoins saisonniers non couverts dans l'agriculture et le tourisme, se conjuguent pour expliquer ce basculement du positionnement géostratégique de la Grèce. Sans en être les bénéficiaires prioritaires - les villes, grandes et petites, ont été, c'est bien connu, les bases principales de réinvestissement immobilier et de services banals pour les capitaux rapatriés par les travailleurs migrants, et plus récemment, les Grecs Pontiques, originaires des contrées russes de la Mer Noire, ont préféré massivement une installation à Athènes plutôt que dans les villages qui leur étaient préparés en Thrace les campagnes ont aussi profité d'installations plus ou moins définitives. Cueillette des produits agricoles, construction, main-d'œuvre touristique non qualifiée dans la restauration et l'hôtellerie, justifient ces apports démographiques que la statistique ignore, que la compatibilité nationale sous-estime, mais sans lesquels la vie économique de l'espace rural serait impossible, en tout cas moins prospère.

21 Suffisent-ils pour autant à compenser le handicap structurel majeur de toute terre d'émigration: le vieillissement démographique? Même si dans certains villages des Cyclades, on se félicite, à demi-mots gênés, que le maintien de l'école locale soit justifié par la présence d'enfants... albanais, on peut en douter. Les pyramides d'âges, établies toujours sur la seule base disponible des statistiques officielles, montrent bien le clivage des deux Grèces. En 1991, les plus de 45 ans représentaient plus de la moitié des actifs dans les zones rurales, et même 53 \% parmi les femmes actives, contre respectivement $41 \%$ et $33 \%$ pour l'ensemble de la Grèce. La véritable menace des campagnes grecques, et spécialement de leurs activités agricoles, n'est pas économique, mais démographique. Une enquête personnelle, menée en Messara crétoise à la fin des années quatre-vingt, montrait déjà que les choix stratégiques dans l'orientation des exploitations modernes n'étaient pas conditionnés par un calcul de rentabilité économique, mais par l'âge des exploitants. Les plus jeunes choisissaient des cultures souvent hors sol, sous serres. Les plus anciens leur préféraient des olivettes irriguées, moins exigeantes en soins et en présence, mais sans être à l'époque assurés de leur succession. L'exode rural n'est pas à l'ordre du jour, mais la déprise agricole menace toujours, politique agricole commune ou pas.

Enfin, il faut de la même manière considérer avec circonspection la conviction univoque d'une condamnation sans appel des populations vivant dans les zones géographiques accidentées (tableau 3, cartes 5 et 6). Si l'ensemble de la croissance démographique du pays est désormais concentré dans les régions d'altitude les plus basses (+ 350000 habitants dans les zones de plaines et +150000 dans les zones semimontagneuses entre 1981 et 1991), le volume de la population réputée "montagnarde " ne varie pas au cours de la dernière période intercensitaire : 940000 personnes en 1981, comme en 1991. Certes, il faut faire la part du caractère artificiel des délimitations statistiques. Et les retours fictifs le jour du recensement sont particulièrement signalés 
dans les villages de montagne. Mais il faut aussi tenir compte des modifications des genres de vie. Partout en Grèce, simultanément à la littoralisation de la population, la pente et le versant retrouvent leurs attraits séculaires, en même temps que la motorisation rend les montées aisées, que les engins de nivellement façonnent les terrasses pour des constructions modernes ou même que les cultures arbustives organisées se plaisent mieux sur des pans de collines exposées au soleil, à l'abri des brumes et même des gelées hivernales. La vieille logique de la descente de l'habitat et de l'économie rurale recule devant le resurgissement d'attitudes plus anciennement ancrées, que les mœurs écologiques et la volonté de jouir de vues étendues remettent au goût du jour. Battements millénaires de la nappe de l'œekoumène méditerranéen entre montagne et plaine ? En tout cas, la culture pèse ici plus lourd que l'agriculture.

Carte 5 : Répartition de la population grecque en 1991 (zones de plaines, semi-montagneuses et montagneuses)

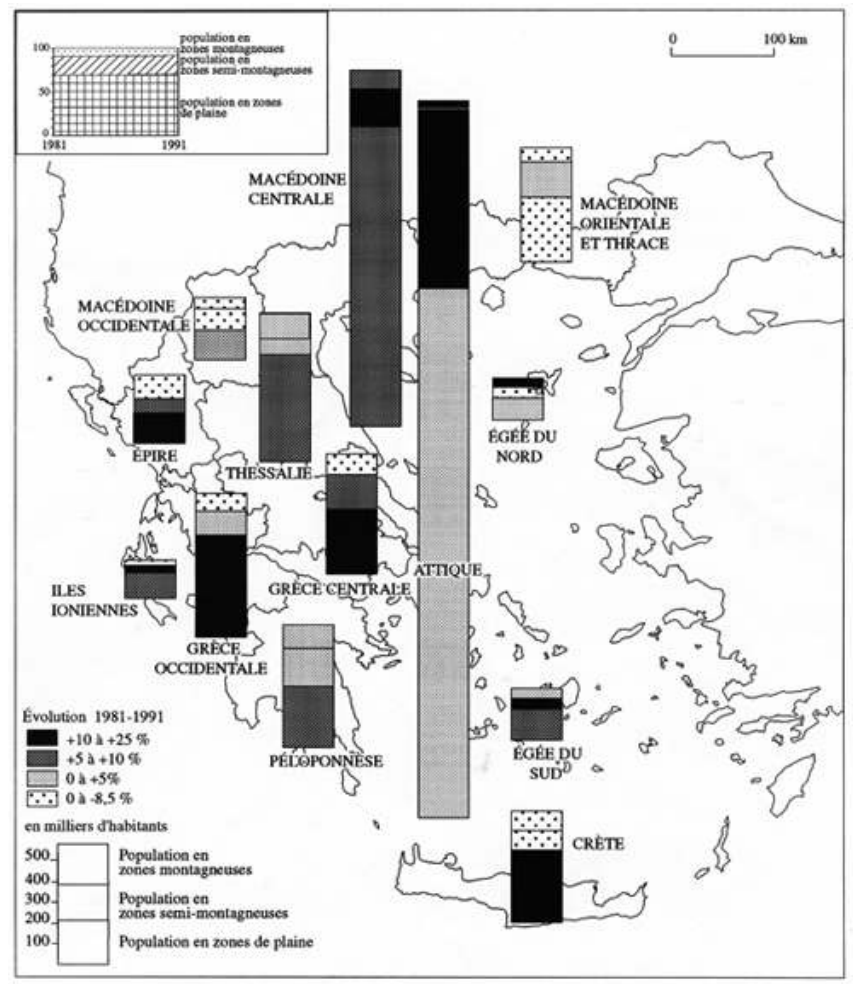




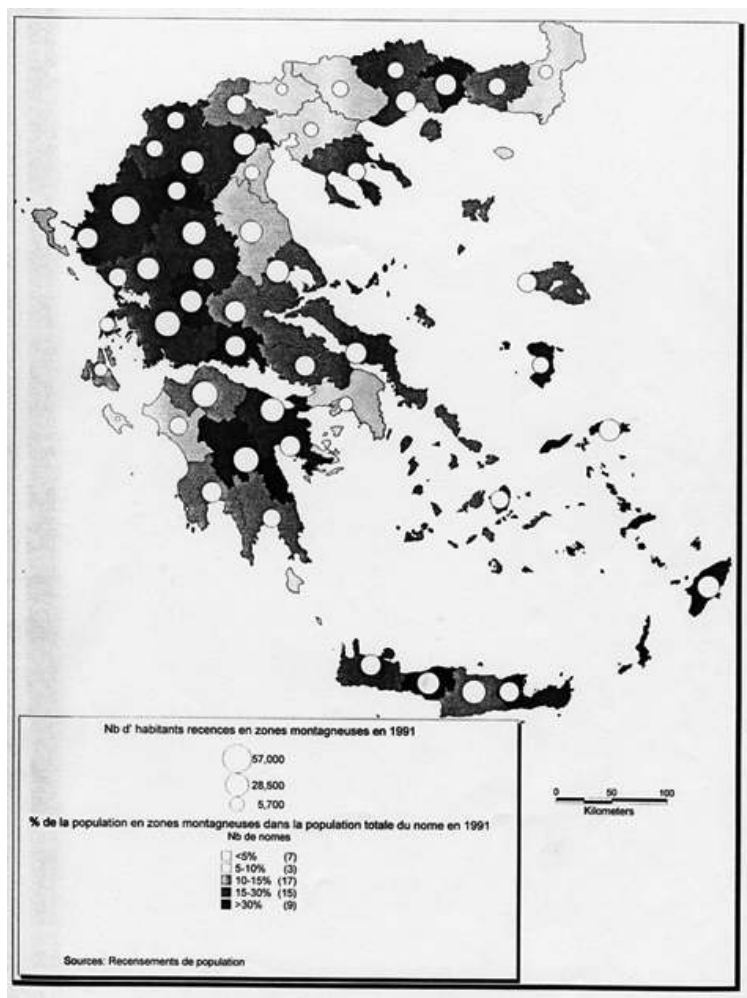

Des campagnes qui ne sont plus majoritairement agricoles

La clef, sans doute, de compréhension des paradoxes de l'espace rural grec et de pronostic sur son avenir, réside dans la prise de conscience de ses évolutions économiques et sociales récentes. On peut les résumer par une formule choc: il n'y a plus d'exode rural, mais l'exode agricole s'amplifie. Au-delà vraisemblablement des données fournies par les statistiques officielles. Alors que la population rurale ne connaît, on le sait, qu'une faible décroissance de 50000 habitants, la population active agricole a régressé de 300000 personnes entre 1981 et 1991, dont 260000 dans les zones rurales. 110000 d'entre elles étaient des chefs d'exploitation, et 120000 des femmes non rémunérées travaillant sur l'exploitation de leurs proches. Ces simples approximations grossières suffiraient à démontrer l'ampleur des mutations professionnelles, sociales, collectives et individuelles, plus que géographiques, dans les campagnes grecques. Elles touchent l'équilibre des activités rurales à l'échelle du pays, des régions, des noyaux de peuplement villageois et de la cellule familiale.

De façon plus détaillée, l'agriculture, étendue à l'élevage et à la pêche, ne représente plus que $46 \%$ des actifs recensés dans les zones rurales en 1991 (tableau 4, fig. 1). Désormais, les services, le commerce et l'hôtellerie-restauration, l'industrie et la construction réunis, y font presque jeu égal avec les activités agricoles et rurales classiques. Alors que la population active rurale totale a été amputée de 100000 unités (indice 92 pour une base de 100 en 1981), l'agriculture a perdu un tiers de ses effectifs de début de période intercensitaire, tandis que ceux des services, du commerce et de l'hôtellerie et de la distribution d'énergie et d'eau, ont été presque multipliés par deux. 


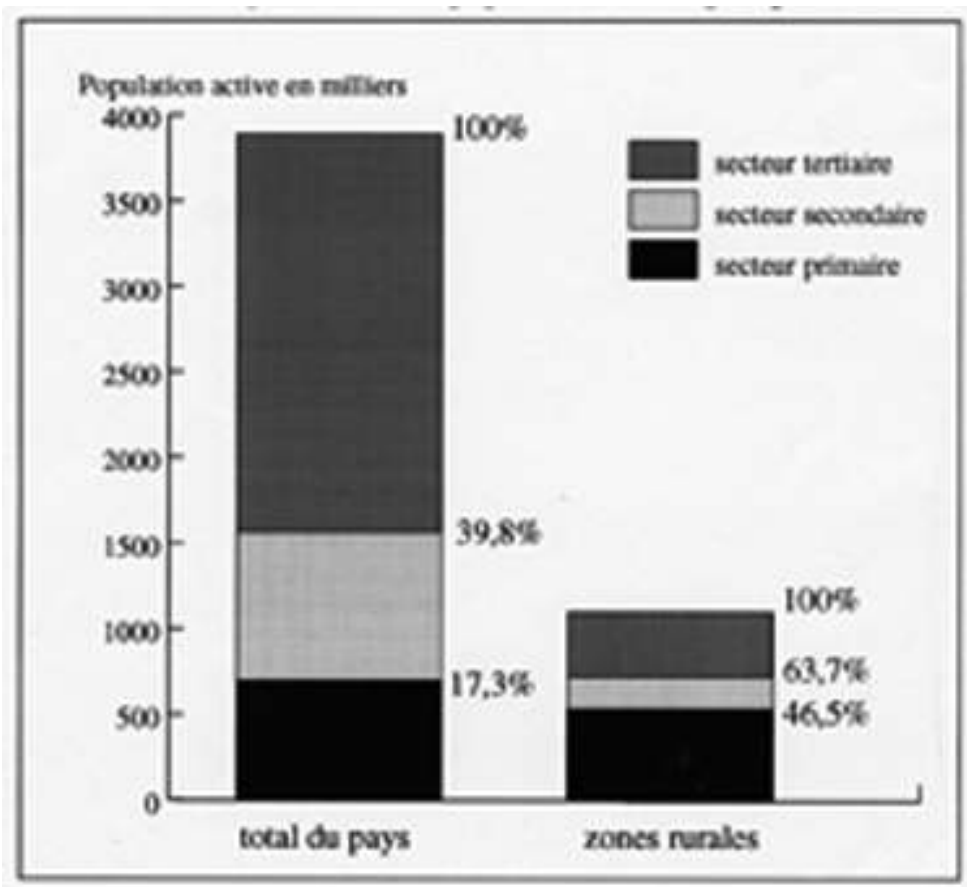

Figure 1 : Structure comparative de la population active grecque en 1991

La répartition par tranches d'âges et par sexe permet d'affiner ces processus du changement social et professionnel dans les campagnes. Ils apparaissent toucher toutes les strates de la société rurale, mais de façon diversifiée. L'érosion des activités agricoles est plus sensible aux deux bouts de la chaîne démographique, chez les plus jeunes et chez les plus âgés, et plus rapide chez les femmes que chez les hommes. Elle affecte donc les modalités de renouvellement et de fonctionnement du personnel agricole. D'une part, les départs à la retraite d'agriculteurs âgés ont été plus amples que la déperdition moyenne, et les nouvelles générations plus abondantes à se tourner vers des professions non directement liées à l'agriculture. D'autre part, les femmes, qui fournissaient de forts contingents de main-d'œuvre dans les exploitations familiales, ont vu proportionnellement leurs effectifs fondre plus vite, passant en dix ans de $31 \%$ de la population active agricole à $27 \%$. Dans une certaine mesure, ces évolutions inégales ont renforcé la part des agriculteurs dans la force de l'âge; la population active agricole âgée de 20 à 44 ans passe de 33 à $37 \%$ du total de 1981 à 1991. Mais à terme, si les nouvelles générations continuent sur la même tendance, le déséquilibre de la population active agricole est inévitable : l'évolution des dernières décennies n'aura fait que retarder les effets du vieillissement généralisé des zones rurales.

À l'inverse de l'agriculture, le commerce, l'hôtellerie et l'ensemble des services ont gagné plus de 100000 actifs pendant la dernière période intercensitaire et représentent désormais plus du quart des actifs des zones rurales. Cette transformation est évidemment à mettre au compte inégal de l'équipement et du désenclavement des zones rurales, mais surtout du développement du tourisme. L'évolution des catégories socioprofessionnelles réserve d'autres surprises, même si elle renforce la constatation d'une urbanisation rapide des campagnes helléniques (tableau 5, fig. 2). La Grèce est certainement un des rares pays où la progression des groupes sociaux réputés liés aux activités tertiaires (professions intellectuelles et libérales, cadres administratifs, 
employés de bureau et des services, commerçants et vendeurs) a été beaucoup plus vive dans les zones rurales que dans les villes, tandis que la population active agricole diminuait un peu plus rapidement dans les villages que dans les agglomérations urbaines! Pour apprécier ces évaluations à leur juste valeur, il faut néanmoins prendre garde aux masses absolues mises en cause (les professions intellectuelles et libérales recensées dans les campagnes ne représentent en 1991 que $12 \%$ du total de la Grèce) et surtout à une statistique liée au lieu de résidence, et non au lieu d'emploi : beaucoup de ces actifs du secteur tertiaire exercent leur métier dans les petites villes ou les agglomérations moyennes, tout comme les 56000 agriculteurs comptabilisés dans les villes n'y ont pas pour la plupart le siège de leur exploitation. La remarque méthodologique renforce cependant le complexe ruralo-urbain déjà mis en lumière.

Là encore, l'examen des structures d'âge du changement professionnel montre la rapidité des transferts parmi les plus jeunes. Dans les zones rurales, les agriculteurs de 20 à 44 ans, dont on a pourtant souligné la meilleure résistance, perdent en dix ans 75000 actifs, gagnés exactement en termes de bilan par les activités tertiaires. Comme à l'accoutumée, les rythmes du changement ont été encore plus rapides pour les jeunes femmes. L'analyse menée sur les statuts professionnels permet en outre de suivre dans cette mutation de l'économie les permanences des spécificités de la société grecque (tableau 6). Malgré sa progression sensible au cours de la dernière période intercensitaire, le salariat reste toujours relativement faible en Grèce, ce qui est normal dans les campagnes, en raison de la présence des exploitants agricoles, ce qui est plus original en ville ( $49 \%$ dans l'ensemble du pays, $62 \%$ dans les zones urbaines). Dans les transformations actuelles des structures professionnelles, il faut souligner le maintien de l'initiative individuelle et familiale. En dix ans, le nombre d'employeurs est multiplié par 2,5 dans la moyenne nationale, par 3,5 dans les campagnes. Et la diminution statistique des travailleurs indépendants n'est due qu'aux catégories agricoles. Dans les autres secteurs d'activité, on relève au contraire une forte progression de ce statut: dans les seules zones rurales, il gagne 16000 postes de travail dans le commerce et l'hôtellerie, 10000 dans les services (tableaux 7 et 8). 


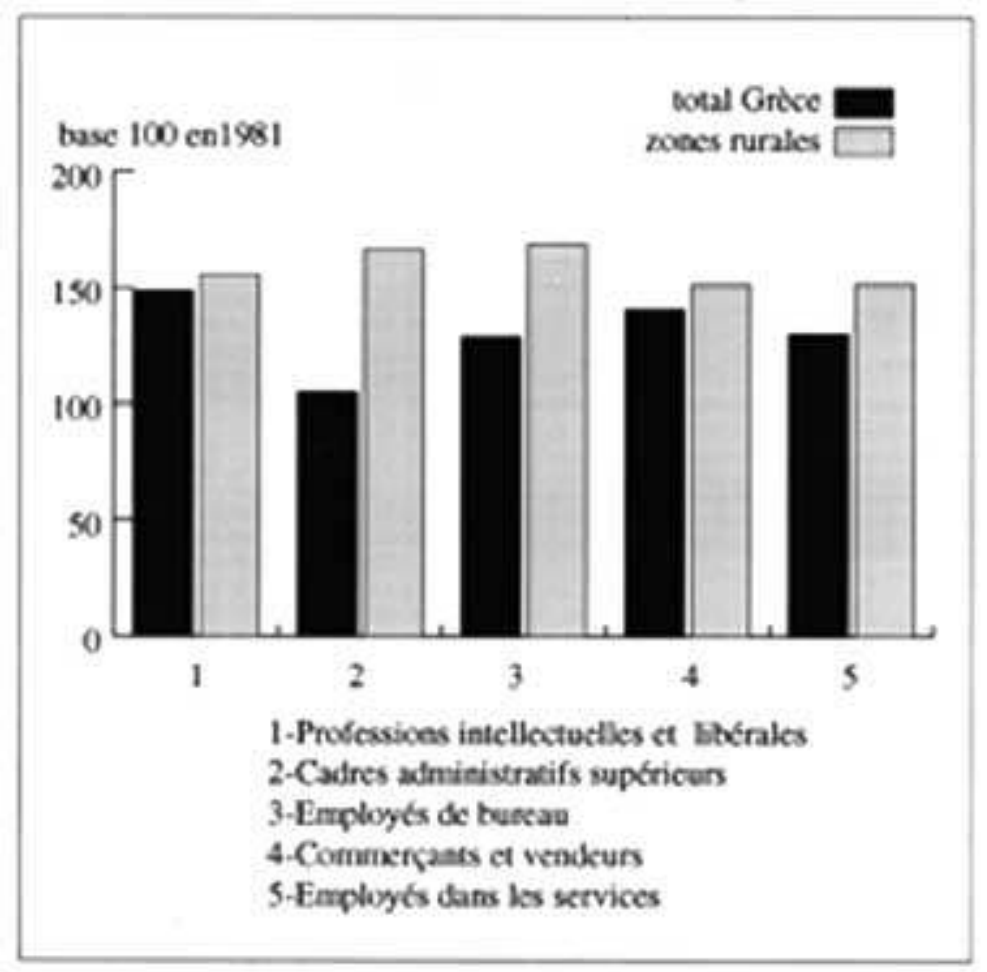

Figure 2 : Progression comparée des professions tertiaires en Grèce et dans les zones rurales (1981-1991) inctivité, habituelle en ville, où elle permet aux citadins de maintenir un niveau de vie acceptable, malgré la relative faiblesse des rémunérations unitaires, et très commune à la campagne. Ainsi, selon le recensement agricole de 1991, un chef d'exploitation agricole passerait 139 jours sur son exploitation, travaillerait 6 jours sur d'autres exploitations, mais ne consacrerait pas moins de 45 jours à des activités autres que l'agriculture. Pour les autres membres de la famille travaillant sur l'exploitation, les nombres respectifs sont 116,5 et 26 jours. On imagine aisément ce que ces moyennes cachent, et révèlent en même temps, de réalités diverses: actif rural exerçant véritablement plusieurs occupations (agriculteur et gestionnaire d'une petite 
unité commerciale ou touristique) au fil de la journée, ou plutôt des saisons, ou actifs d'une même famille, classée agricole en raison de la fluidité des critères statistiques et de la distribution réelle au sein d'une même exploitation d'actifs exerçant des professions dans plusieurs secteurs d'activités (fille employée dans les services administratifs d'une petite ville, fils salarié dans les transports ou chauffeur de taxi à son compte, se retrouvant évidemment chaque soir à la même table sous le toit familial). Cette pluriactivité rurale, qui pourrait apparaître comme un élément de faiblesse pour des entreprises agricoles performantes, est en fait une extraordinaire assurance contre les retournements de la conjoncture... et des politiques, nationales ou communautaires.

Il faudrait pouvoir nuancer ce tableau rapidement brossé par des différenciations régionales. Les statistiques aisément disponibles n'ont pas permis de le faire. Mais il est loisible d'avancer que, si les évolutions retracées sont générales dans le territoire rural de la Grèce, elles sont certainement plus affirmées dans les zones périurbaines, au sens large du terme, et dans les régions de tourisme balnéaire (littoral et îles), où les opportunités d'occupations non agricoles et de pluriactivité sont innombrables (construction, commerce, hôtellerie, restauration, etc.). Mais il ne faut pas oublier que dans les zones de montagnes, comme dans les régions de grandes cultures, les villes petites et moyennes ne sont jamais loin, et que l'élévation des revenus agricoles a multiplié ici les besoins, les désirs, et leur satisfaction. C'est terminer ce panorama par un aperçu du désenclavement de la campagne grecque.

Un espace rural desservi par des infrastructures ramifiées et un réseau urbain dense

31 À cet égard, une vue rapide de la modernisation réelle et projetée dans la société et l'espace grecs, met toujours l'accent soit sur de minuscules transformations du décor familier des villes et des villages (pavage somptuaire d'une rue, d'un lieu public restreint, rendu piétonnier, réfection grandiloquente du monument aux morts, soudain pompeusement rebaptisé dans nombre de bourgades "place des héros»), soit, plus souvent encore, pour les observateurs internationaux avides de chiffres, sur de grands travaux d'infrastructures et des chantiers de travaux publics spectaculaires, nationaux ou urbains. Ainsi, on le sait bien, depuis de nombreuses années, la construction du métro d'Athènes, la reconstitution d'une voie Egnatia coupant la péninsule balkanique et unissant les rivages de la mer ionienne (Igouménitsa) au golfe thermaïque (Thessalonique) et au-delà à la Thrace et à l'Asie Mineure, l'amélioration des télécommunications, notamment par la digitalisation des réseaux, l'édification d'une base énergétique plus souple et moins coûteuse fondée sur le gaz naturel, mobilisent la majeure partie des fonds d'investissements, notamment communautaires, et de l'attention de l'opinion et des spécialistes. Il n'est pas assuré que cette myopie de promeneur et cette presbytie d'expert rendent véritablement compte des transformations profondes et de l'intégration unificatrice du territoire grec.

Sans en faire une critique, ni un recensement systématique, on demeure toutefois sceptique sur les résultats attendus de cette cartographie à la mode d'axes et de pôles, bientôt transformés en réalités, sous l'effet des écus, maintenant des euros, et de la puissance technique des grandes entreprises de travaux publics. Que la nouvelle voie Egnatia, déjà citée, élargisse les débouchés de la Macédoine agricole, on est prêt à l'admettre. Qu'elle reconstitue du coup un courant d'échange historique vers Brindisi et Bari, sous raison qu'il faudrait unir deux pôles déprimés de l'Europe - la Méditerranée centre-orientale et le Mezzogiorno -, on devient plus dubitatif, quand on connaît la 
pente " naturelle » actuelle du trafic vers Ancône et les régions riches de l'Europe, et l'investissement en gros porteurs maritimes rapides. De la même façon, la programmation d'un axe routier Igouménitsa-Kalamata destiné à contrebalancer à l'ouest l'existence de la branche fertile de l'espace grec, Thessalonique-Athènes, peut rappeler la politique française des «arcs » - atlantique, méditerranéen. Élide mise à part, on ne voit pas bien ce que cette autoroute des régions de "l'envers", pour paraphraser les dissymétries du Japon, pourra apporter aux zones rurales concernées, sinon relier plus rapidement des centres urbains, souvent isolés au milieu de campagnes plus ou moins vides, Iannina, Arta, Agrinion. De même, il est douteux que la plaine thessalienne ait attendu pour son désenclavement l'hypothétique amélioration de la desserte ferroviaire Kalambaka-Kozani, ou que la Thrace ait une patience identique pour la voie ferrée Kavala-Xanthe.

En fait, au-delà des grands axes routiers, dont l'importance nationale et internationale est indéniable, et dont l'équipement et la sécurité se sont considérablement enrichis au cours des dernières années, notamment aux sorties d'Athènes, c'est bien la dissémination très diffuse des améliorations sur le réseau des routes interurbaines, voire secondaires, qui est la grande nouveauté dans les campagnes grecques depuis deux décennies. Avec l'essor spectaculaire et plus récent du téléphone mobile, il faut comprendre ce que cette révolution de l'asphalte, du franchissement sûr et permanent de petites ravines, qui peuvent soudain se transformer en torrents, a signifié de désenclavement profond pour les espaces ruraux, les hommes et leurs productions. C'est brutalement se trouver, en toutes circonstances, relié moins au monde, à Athènes ou à Thessalonique, qu'à l'univers social, économique et technique, de la petite ville proche, et guère plus lointainement de la capitale provinciale. Au XIXe siècle, dans les campagnes françaises, le débat a pu être ouvert sur les conséquences du développement du réseau ferré sur l'exode rural, accélération de la vitesse de l'émigration ou frein par l'arrivée du progrès. Dans la Grèce contemporaine, la question n'est pas permise. Les routes de terre, l'âne et le tracteur des années soixante, promettaient la poursuite de l'hémorragie rurale. Le goudron et la voiture ont permis la mobilité résidentielle et professionnelle, qui a maintenu la substance démographique - sinon la vitalité, eu égard au vieillissement des structures d'âges déjà amplement souligné - en l'état dans les campagnes grecques. En raison de l'ampleur sans précédent, en Grèce comme ailleurs, et de la rapidité des transformations des communications, matérielles et immatérielles, un retournement de tendance est peu probable.

Ce désenclavement généralisé de l'espace rural grec a été rendu plus facile encore par la relative proximité dans toutes les régions de petites ou de moyennes villes. Il ne s'agit pas d'affirmer ici, au contraire, l'existence en Grèce d'un réseau urbain strictement hiérarchisé, qui constituerait en outre mécaniquement les bases matérielles de "pôles décentralisés de développement". Mais il faut souligner un paradoxe de l'évolution du territoire hellénique. On assiste simultanément à la contraction, toujours remarquée, de l'espace utile et des dynamismes urbains les plus vifs et surtout les plus massivement visibles dans le croissant Thessalonique-AthènesPatras, et au maintien, et même à la progression de petites villes, plus isolées au milieu de régions en déprise démographique et économique (carte 7). Pour s'en tenir à des villes de moins de 30000 habitants, c'est le cas de Ptolémaïs, Iannitsa, Edessa, Kilkis, en 
Macédoine, de Tripolis, Argos, Sparte, Amalias, Nauplie, dans le Péloponnèse, de Prévéza en Épire.

Carte 7 : Tendances d'évolution des agglomérations grecques de plus de 10000 habitants en 1991 (1961-1991)

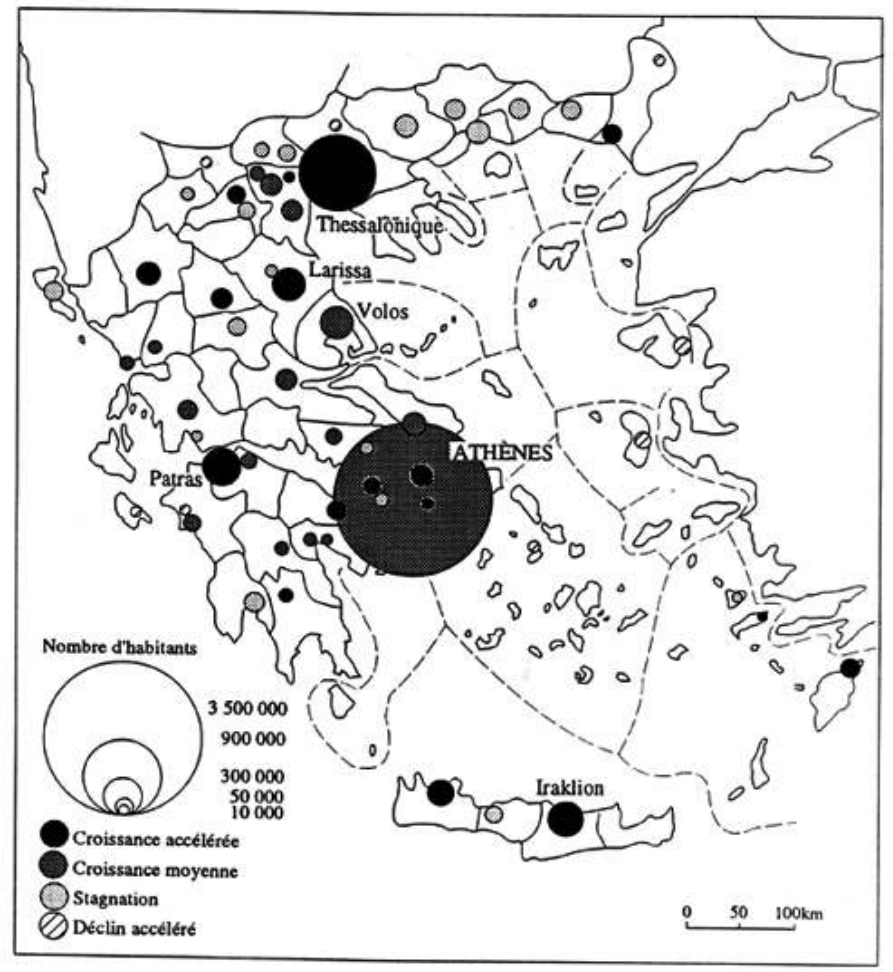

En fait, au-delà de l'énumération, la Grèce démontre à la fois sa conformité par rapport au modèle européen de transformation territoriale et le maintien de spécificités historiques ou culturelles. Une recherche comparative entreprise sur l'évolution démographique des régions et des villes en France et en Grèce au cours des trois dernières décennies montre bien similitudes et différences. Le même modèle spatial s'impose, qui traverse les trois dernières décennies, mais s'est en fait mis en place bien avant: la rupture de l'uniformité du territoire sous l'effet des dynamismes démographiques. De 1962 à 1990, plus de la moitié des départements français ont connu une croissance supérieure à la moyenne nationale. En Grèce, c'est près de $40 \%$ des unités de même type, les nomes, qui sont dans une situation identique entre 1961 et 1991. La régularité statistique entre les deux pays tend même à s'uniformiser au cours de la dernière période intercensitaire (1982-1990, 1981-1991 respectivement), en accentuant l'ampleur des zones de déprise démographique : $62 \%$ des départements français, 59 \% des nomes grecs sont comptés en stagnation ou en déclin accéléré. Dans les deux pays, les régions de croissance continue s'appuient sur les grandes villes du dispositif et s'élargissent autour d'elles. La diffusion urbaine contemporaine a en même temps dissocié l'espace national et recomposé le territoire en masses spatiales homogènes. À cause des dynamismes urbains, l'espace utile s'est à la fois rétracté dans la nation, et diffusé dans une minorité de régions sélectives.

Pourtant, une différence remarquable caractérise ce modèle spatial: une plus large diffusion de la croissance démographique en France, une urbanisation beaucoup plus concentrée en Grèce. Ici, non seulement les cas de croissance accélérée sont plus limités 
en nombre, mais strictement restreints aux abords immédiats des plus grandes villes : Athènes, Thessalonique, Patras, dans une moindre mesure, Iraklion et Volos. Il y a là incontestablement un effet spatial de la concentration urbaine, qui rappelle le moindre développement économique. Ces organisations géographiques du territoire se doublent d'une évolution historique des dynamismes démographiques. Dans les deux pays, la croissance urbaine se ralentit, sous l'effet conjugué de la chute de la fécondité et des dilutions périphériques des agglomérations, qui noient les villes dans leurs auréoles périurbaines. Pour les raisons déjà évoquées, le phénomène est moins net en Grèce qu'en France : sur les 110 villes de plus de 50000 habitants étudiées, 48 connaissaient une croissance accélérée par rapport à la moyenne nationale de 1962 à 1982, elles ne sont plus que 14 de 1982 à 1990 ; sur 62 villes grecques de plus de 10000 habitants la régression est moins nette : 26 villes en croissance accélérée de 1961 à 1981, encore 21 de 1981 à 1991.

Mais l'essentiel n'est pas là. La véritable dimension du changement est le coup d'arrêt apporté en France, au cours de la dernière décennie, à la progression des villes petites et moyennes qui avait marqué pendant la période précédente une réelle déhiérarchisation, voire démocratisation, du modèle urbain. Le tableau suivant montre que la Grèce est encore sur ce modèle.

Évolution typologique des villes en France et en Grèce

(nombres absolus de cas observés)

Les plus grandes villes confirment leur meilleure résistance dans la crise

\begin{tabular}{|l||l|l||l|l|}
\hline \hline & $\begin{array}{l}\text { En France sur } 17 \text { agglomérations } \\
\text { de plus de } 300000 \text { hab. }\end{array}$ & $\begin{array}{l}\text { En Grèce sur } 12 \text { villes } \\
\text { de plus de } 50000 \text { hab. }\end{array}$ \\
\hline \hline Type d'évolution démographique & $1962-1982$ & $1982-1990$ & $1961-1981$ & $1981-1991$ \\
\hline \hline Croissance accélérée & 6 & 2 & 9 \\
\hline \hline Déclin accéléré & 3 & 4 & 0 \\
\hline
\end{tabular}

Les villes petites et moyennes poursuivent leur dynamisme en Grèce

\begin{tabular}{|l||l|l|l|l|}
\hline \hline & $\begin{array}{l}\text { En France sur 53 agglomérations de } \\
50000\end{array}$ & $\begin{array}{l}\text { En Grèce sur 36 villes } \\
\text { à } 100000 \text { hab. }\end{array}$ \\
30 000 hab. & 1000 à \\
\hline \hline $\begin{array}{l}\text { Type d'évolution } \\
\text { démographique }\end{array}$ & $1962-1982$ & $1982-1990$ & $1961-1981$ & $1981-1991$ \\
\hline \hline Croissance accélérée & 24 & 7 & 10 \\
\hline \hline Déclin accéléré & 2 & 20 & 15 \\
\hline
\end{tabular}


38

erprétation de ces résultats différenciés entre la France et la Grèce n'est pas aisée.

En fait, l'intérêt est bien de suivre, dans l'espace et dans le temps, les permanences et les variations de deux cycles de l'histoire urbaine que notre période superpose et collisionne à l'envi : un cycle de diffusion qui favorise les extensions périphériques dans les auréoles périurbaines des très grandes agglomérations et, plus largement, les dilutions du système spatial dans les plus petites unités urbaines et un cycle d'innovation qui reconcentre le dispositif de commande économique, de création et même de consommation culturelles. Dans cette hypothèse, la crise économique, ou plutôt, la profonde mutation du système productif et des stratifications sociales, entraîne une nouvelle concentration, dans les plus grandes villes, de la modernité et de la créativité, sans qu'elle s'accompagne nécessairement de fortes croissances démographiques. Avec ses hypertrophies urbaines et ses diffusions citadines simultanées, la Grèce est manifestement en retard d'un cycle... ou sur un itinéraire différent. Selon la réponse, la crainte d'un regain de croissance dans les très grandes villes serait justifiée, ou au contraire l'appui sur le semis des villes secondaires conforté.

exemple amusant illustrera cette diffusion de la modernité dans l'espace grec. Tout récemment, une chaîne de distribution de petits équipements de cuisine assez luxueux, et quelquefois raffinés, même si le goût en est discutable, s'est implantée en Grèce sous le nom de Cook-Shop. Sur 16 rayons ouverts en général dans des grandes surfaces, la moitié est située dans l'agglomération athénienne, pas nécessairement d'ailleurs dans les quartiers les plus aisés, mais là où le dynamisme des couches moyennes supérieures a été particulièrement vif au cours des dernières années (Glyphada, Amaroussion). Sur les huit implantations relevées en province, seules les boutiques de Santorin, et dans une moindre mesure de Céphallonie, peuvent témoigner des attitudes et des moyens des nouveaux riches de la manne touristique. Les six autres cas sont tous localisés dans des centres de régions agricoles prospères, mais pas statistiquement dans les plus grandes villes du pays: Iraklion et Larissa, qui n'atteignent pas 150000 habitants, Serrès, Lamia, Verria et Drama, qui ne dépassent 50000 habitants. L'anecdote est parlante: ni la hiérarchie urbaine, ni la hiérarchie sociale ne sont strictement respectées; et l'enrichissement agricole se résout plus en consommation ostentatoire qu'en investissement productif. De quoi faire réfléchir sur les moteurs de l'évolution dans les campagnes grecques.

Au terme de ce périple quelque peu cavalier dans le temps et l'espace des organisations de la Grèce contemporaine, il n'est pas inutile d'en proposer une leçon plus synthétique. La première est certainement la fluidité, plus grande qu'ailleurs, des distinctions classiques entre villes et campagnes. L'incertitude tient moins aux formes bâties ou même aux classiques discontinuités territoriales qu'à l'identité croissante des modes de vie et, depuis peu, au renforcement des attaches entre ruraux et urbains. L'exode des villages était sans doute trop récent en Grèce, les distances géographiques insuffisamment importantes entre les régions, pour que se perde la mémoire, entre gens et générations, des liens tissés entre bourgs, petites villes et grandes agglomérations. L'accélération des mobilités, la route, l'avion et le ferry rapide ont fait le reste, multipliant les contacts, les voyages, les séjours saisonniers, et rendant plus imprécise que dans bien des pays plus développés la notion même de résidence principale. Il faut y songer dans l'exploitation des recensements actuels et à venir, 
plutôt que d'accuser toujours la fiabilité des statistiques et la duplicité des politiques. C'est la réalité qui est double ou triple.

41 La seconde indication est plus économique que géographique. Elle est inspirée par la poussée spectaculaire des activités tertiaires dans les campagnes grecques. Que la présence spécifique du tourisme l'amplifie et que les mobilités résidentielles déjà évoquées la justifient en partie n'enlèvent rien à l'ubiquité du mécanisme. La tertiarisation de l'économie n'a pas seulement transformé le procès de production dans les espaces agricoles, en donnant la priorité à l'agro-alimentaire et à la réglementation dans l'élaboration des produits de la terre. Elle a durablement retourné une logique historique séculaire. La révolution industrielle avait concentré la fabrication dans la ville. La révolution tertiaire polarise tout à la fois l'invention et la gestion de l'activité dans les métropoles et la diffuse dans les campagnes.

Enfin, pour illustrer la continuité des cultures et des sociabilités dans l'expression de ces tendances universelles, incontestablement la Grèce témoigne de la persistance et de la vitalité des organisations familiales et de leurs liaisons avec le dynamisme de la petite entreprise. Il y a là une coïncidence qui n'est pas sans rappeler, dans un tout autre contexte, le " miracle » de la troisième Italie. Loin d'être un archaïsme, c'est un atout pour le pays. Mais l'alchimie en est incertaine, et sans doute non transférable.

\section{BIBLIOGRAPHIE}

Beopoulos Nikos, Damianakos Stathis, « Grèce : le cache-cache entre la modernité et la tradition ", in Vers un rural post-industriel, rural et environnement dans huit pays européens (dir. Marcel Jollivet, Paris, L'Harmattan, 1997, p. 177-231.

Burgel Guy, «Vingt ans de changements ruraux en Messara crétoise », Meredies, n 9-10, janvierdécembre 1989, p. 103-112.

Burgel Guy et alii, La CEE méditerranéenne, (notamment le chapitre rédigé par Michel Drain, « Agricultures et sociétés rurales »), Paris, Sedes, 1990.

Burgel Guy, «Trois décennies de démographie dans un village crétois », Revue grecque des sciences sociales, Athènes, Centre national de recherches sociales, nº spécial 79 A, 1990, p. 57-71.

Burgel Guy, «Le périurbain grec : entre banal et spécifique », Méditerranée, n 1, 2/1993, p. 45-48. Burgel Galia et Guy (dir.), « Pobia, étude d'un village crétois », Architectures, Laboratoire de géographie urbaine, Nanterre, 1994, 124 p.

Burgel Guy, Anastassiadis Aghis, « Dynamiques urbaines comparées en France et en Grèce », Petites et grandes villes du Bassin Méditerranéen, Études autour de l'œuvre d'Etienne Dalmasso, École française de Rome, 1998, p. 493-504.

Deslondes Olivier, «L'évolution de la population grecque (1981-1991) : vers le 'modèle' européen ?", Méditerranée, n 1, 2/1995, p. 53-62. 
« Development prospects of the central Mediterranean regions (Mezzogiorno, Greece) », European Commission, Regional Development Studies, Brussels, Luxembourg, 1995, 281 p.

Kassimis Charalambos and Louloudis Léonidas (eds): « Rural Greece, Fragile Structures and New Realities, Sociologica Ruralis ", Volume 37, Number 2, August 1997, Journal of the European Society for Rural Sociology, Agricultural University, Wageningen, The Netherlands.

Lambiri-Dimaki I., Kypiazi N. (eds), La société grecque à la fin du XXe siècle, Athènes, Papazissi, 1995 (en grec).

Recensements de population 1961, 1971, 1981, 1991, Office national statistique de Grèce.

Recensements agricoles, 1981, 1991, Office national statistique de Grèce.

«La situation de l'agriculture dans l'Union européenne », Rapport 1996, Commission européenne, Bruxelles, Luxembourg, 1997.

\section{RÉSUMÉS}

En trente ans, les disparités géographiques du territoire grec se sont accentuées. La montagne, le monde insulaire, les grandes régions agricoles de plaine, les zones périurbaines des grandes agglomérations, composent une typologie simple, mais assez éclairante. Malgré ces divisions, l'exode rural est stoppé depuis les années quatre-vingt et surtout les campagnes ne sont plus majoritairement agricoles. La progression des occupations tertiaires, la mobilité quotidienne et résidentielle des populations, la pluriactivité, diversifient les genres de vie et constituent dans la société et l'espace grec un complexe ruralo-urbain original. Motorisation des ménages, infrastructures routières ramifiées, réseau dense des petites villes, ne sont pas étrangers à ces traits spécifiques, où les sociabilités méditerranéennes retrouvent leurs droits.

Over the past thirty years, geographical disparities have increased. Areas such as the mountains, the islands, the large farmlands in the plains and the peripheries of large urban agglomerations display a simple typography, but which is telling nonetheless. Despite the differences, rural to urban migration stopped in the 1980s and, most importantly, rural areas no longer remain principally agricultural. The increase in employment in the service sector, in both daily and residential mobility, in the multiplication of activities, has diversified lifestyles and introduced an original rural-urban complex into Greek society and space. The motorisation of households, multiple road infrastructures, dense networks in small towns all contribute to these particular characteristics, in which Mediterranean sociability discovers new life.

\section{INDEX}

Index géographique : Grèce

Mots-clés : Espace

\section{AUTEUR}

GUY BURGEL

Laboratoire de géographie urbaine, UMR LOUEST, Cnrs, université de Paris X-Nanterre 